\title{
Influence of Tracheal stenosis on Vocal Fold Vibration: A case report
}

\author{
Case \\ Report \\ Eman Mostafa, Mohamed Elrabie Ahmed \\ Department of Otorhinolaryngology, Faculty of Medicine, Sohag University, Egypt.
}

\begin{abstract}
Objectives: Patients with laryngotracheal stenosis (LTS) suffer from various problems such as dyspnea, stridor, intolerance of a speaking valve or inability of tracheostomy decannulation. The impact of LTS on the voice has been poorly understood and studied, particularly in adults. There has been no detailed qualitative or quantitative data about voice quality in patients with LTS. This case report presents tracheal stenosis with stridor and dysphonia with abnormal patterns of vocal fold vibration.

Case Report: We report a female patient with post-tracheostomy tracheal stenosis presented with moderate stridor and dysphonia due to an atypical pattern of vibration, which has been reversed after balloon tracheal dilatation using rigid bronchoscopy. The case showed marked improvement after dilatation in respiratory distress, dyspnea and the voice quality as evidenced subjectively by Auditory Perceptual Assessment (APA) and objectively by acoustic analysis.

Conclusion: Tracheal stenosis affects the mechanism of vocal fold vibration, which is reversed after tracheal dilatation with subsequent improvement of vocal fold vibration.
\end{abstract}

Key Words: Dilatation, laryngotracheal, stenosis, voice outcome.

Received: 6 July 2020, Accepted: 31 July 2020

Corresponding Author: Mohammed Elrabie Ahmed, MD, Nasr City, Eastern Avenue, University Street. Tel.: 01226200444, E-mail: dr.mohammedelrabie@gmail.com

ISSN: 2090-0740, November 2020 Vol.21, No.3

\section{INTRODUCTION}

The Endotracheal intubation or tracheostomy are most commonly used to deliver mechanical ventilatory support. Despite technological improvements and skillful patient care, laryngotracheal stenoses still a serious clinical sequalae which make decannulation process difficult. ${ }^{[1]}$

LTS is a partial or complete narrowing of glottic or subglottic or tracheal lumen due to submucosal scar or cartilage collapse. Patients with LTS often suffer from various problems such as dyspnea, stridor, intolerance of a speaking valve or inability to decannulate if a tracheostomy tube is present. ${ }^{[2]}$ LTS treatment has focused on improving the airway caliber to improve dyspnea and stridor. Voice outcomes are less explored. Some studies have reported successful improvement in dyspnea and voice outcomes in children. ${ }^{[3,4]}$ There has been no detailed qualitative or quantitative data about voice quality in those patients.

The impact of LTS on voice has been poorly studied, particularly in adults. ${ }^{[5]}$ It has been noted that the infraglottis shape affects airflow turbulence reaching the vocal folds during phonation. In this case report, tracheal stenosis changed the mechanism of phonation causing the arytenoids to vibrate instead of the vocal folds producing severe harsh dysphonia. Hence, we report a case of post- tracheostomy tracheal stenosis presented with atypical pattern of vibration, which has been reversed after tracheal dilatation using rigid bronchoscopy.

\section{Case report:}

A 20-yr old female married patient with eclampsia developed repeated attacks of convulsions after labour with loss of consciousness. Convulsions attacks was generalized tonic clonic seizures each attack last for 23- minutes. The patient received bolus of magnesium sulfate $1 \mathrm{mg} / \mathrm{kg} / \mathrm{h}$ infusion for 48 hours which increased to double doses when fits was not completely controlled. Diazepam $10 \mathrm{mg}$ intravenously for control of each attacks. After one week, after control of fits medication was discontinued with improvement of convulsion. The patient was diagnosed in ICU as HELLP syndrome because the presence hemolysis evidenced with anemia with reticulocytosis, elevated liver enzymes and low platelet count. The patient was admitted in the ICU and endotracheal intubation was placed for one month. Blood and fresh frozen plasma were transfused appropriately. Computer tomography (CT) brain revealed PRES syndrome (Posterior reversible encephalopathy syndrome). It is a condition caused by reversible subcortical vasogenic edema 
and hypodensity areas of both occipital lobes. Afterwards, tracheostomy was done and decannulated twenty-three days later. The patient was presented in the phoniatric unit in $21^{\text {st }}$ of July 2018 with irregular dysphonia grade III, diplophonia (according to GRBAS scale), low pitched, moderate stridor during exertion (voice video 1) and chocking for fluids, semisolids and solids. Laryngoscopic examination revealed left vocal fold immobility, left arytenoid is leaning forward and both arytenoids are meeting together causing vibration and producing harsh voice with no phonation at the level of the vocal folds. The glottal gap could not be assessed as it is obscured by both arytenoids (video 2). Flexible Endoscopic Examination of Swallowing (FEES) revealed aspiration and penetration of all food consistencies. At September 2018, Swallowing has been improved with normal FEES. Yet, dysphonia persists. Therefore, the patient took five sessions of voice therapy using Smith Accent Method with no improvement.

CT neck was done and revealed 95\% tracheal stenosis at the 5th tracheal ring, grade III (according to MyerCotton grading scale) (Figure 1). Balloon dilatation using rigid bronchoscopy was done on January 2019. Two weeks post-dilatation, roughness of voice has been improved leaving the patient with strained dysphonia grade I, in the presence of persistent vocal fold immobility.

Acoustic analysis was done pre and post dilatation to evaluate the voice outcome and show with quantitative data the difference of the voice quality (Table 1). By comparing the patient's data, remarkably increased fundamental frequency was found indicating higher pitch and marked improvement of the voice quality. Jitter was noticeably reduced with minimal decrease of shimmer and noise to the harmonic ratio.

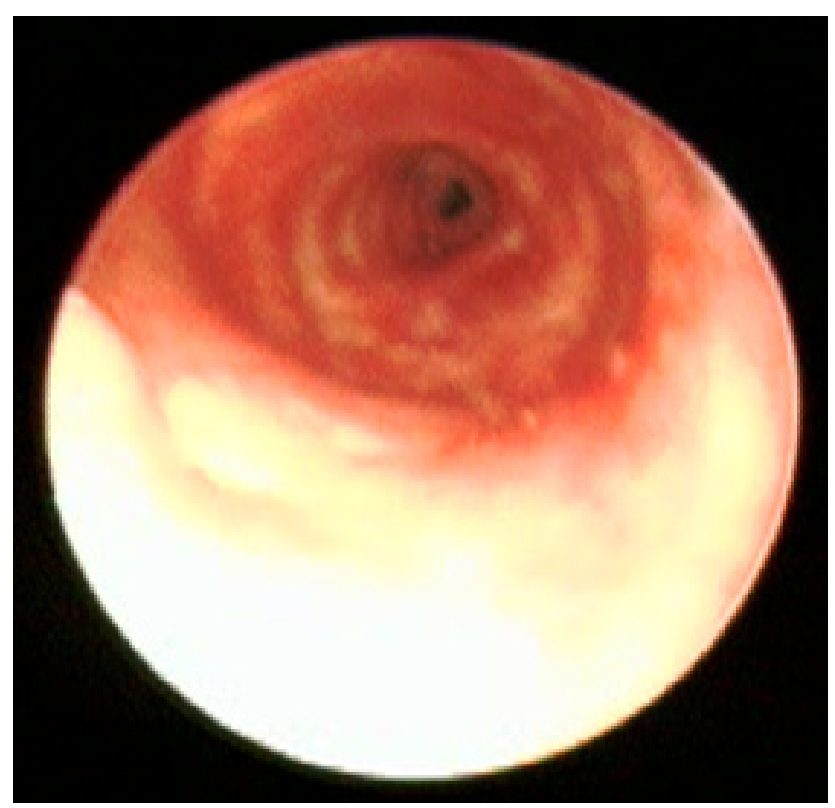

Fig. 1: grade III tracheal stenosis at the fifth tracheal ring
Table 1: Difference of acoustic analysis between pre and post tracheal dilatation.

\begin{tabular}{lcc}
\hline & Pre dilatation & Post dilatation \\
\hline Average FO (HZ) & 89.4 & 255.92 \\
Highest FO (HZ) & 131.25 & 341.06 \\
Lowest FO (HZ) & 71.25 & 187.09 \\
Absolute Jitter & 1263.12 & 229.78 \\
Shimmer & 1.59 & 1.4 \\
Noise to harmonics ratio & 0.472 & 0.324 \\
\hline DISCUSSION & & \\
\hline
\end{tabular}

As the severity of the stenosis increases, the pressure difference across the stenosis increase as well causing increase in the flow resistance. This in turn will decrease the pressure difference across the glottis causing decrease in the glottal resistance and decrease in the driving force on the vocal folds. Smith and Thomson ${ }^{[6]}$ noted that subglottic stenosis affects vocal fold vibration particularly with stenosis severities above $90 \%$. This was observed in our case by laryngoscopic examination showing that the mechanism of phonation was altered. Both arytenoids were vibrating causing arytenoid rattle with no evidence of vocal fold vibration and producing lowpitched irregular voice.

The mucosal wave-like motion of the medial surface is a characteristic feature of vocal fold vibration and has been shown to be an important factor in sustained vocal fold self-oscillation (e.g., Hirano ${ }^{[7]}$, Titze ${ }^{[8]}$, Boessenecker et al. ${ }^{[9]}$. There are many studies investigated the effect of the subglottal shape on vocal fold movement. Smith and Thomson ${ }^{[10]}$ explained that the inferior vocal fold surface angle alters vocal fold stiffness and net flow-solid energy transfer while Li et al. ${ }^{[11]}$ noted that inferior vocal fold surface angle has little effect on the intraglottal pressure distributions that provide the driving force for vocal fold oscillation. Grisel et $a l .{ }^{[12]}$ did not agree with the latter study as they found that changes in the subglottal shape after medialization thyroplasty surgery can significantly increase turbulence through the glottis causing chaotic vocal fold vibrations.

When examining impact of surgery for LTS on voice quality, there were three previous studies, which address dilation instead of transcervical resection/ reconstruction. The first study which was conducted by Bryans et al. ${ }^{[13]}$ utilized a group of 12 patients 
undergoing endoscopic dilation as a control group for patients undergoing resection and documents only postoperative endpoints. The second study was conducted by Hseu et al. ${ }^{[14]}$ over a 10-year interval evaluating 92 patients by Voice Handicap Index (VHIs). The patients showed improvement after dilation. However, dataset limitations prevented the authors from performing statistical analysis. The third study, which was conducted by Hillel et al. ${ }^{[15]}$ evaluated 38 patients with LTS. The authors offered preoperative versus postoperative comparisons and noted improvement of voice quality in single level subglottic or tracheal stenosis after dilatation while the voice quality worsened in half of the cases with multilevel stenosis and in male patients as compared to female patients.

Our case was a female with a single level tracheal stenosis, which showed marked improvement post dilatation in the voice quality as evidenced subjectively by Auditory Perceptual Assessment (APA) and objectively by acoustic analysis. The remaining audible dysphonia (strained grade I) was because of persistent vocal fold immobility. The acoustic analysis is beneficial in describing the voice objectively. In addition, in our case report, we were able to monitor the effectiveness of the dilatation and improvement of voice quality. The fundamental frequency (F0) was remarkably elevated indicating higher pitch and subsequently better voice. Jitter (a measure of frequency instability), in our case, was noticeably reduced knowing that jitter is affected due to lack of control of vibration of the cords. There is minimal decrease in shimmer and noise to the harmonic ratio could be explained possibly due to the persistent vocal fold immobility.

\section{CONCLUSION}

Tracheal stenosis was noted to affect the mechanism of vocal fold vibration causing arytenoid rattle as a compensatory mechanism to produce sound. This compensatory mechanism has been reversed after tracheal dilatation with subsequent improvement of vocal fold vibration.

\section{CONFLICT OF INTEREST}

There are no conflicts of interest.

\section{REFERENCES}

1. Sarper A, Ayten A, Eser I, Ozbudak O, Demircan A. Tracheal stenosis after tracheostomy or intubation: review with special regard to cause and management. Texas Heart Institute Journal. 2005; 32(2):154.)
2. Hatcher JL, Dao AM, Simpson CB. Voice outcomes after endoscopic treatment of laryngotracheal stenosis. Annals of Otology, Rhinology \& Laryngology. 2015 Mar;124(3):235-9.).

3. Zalzal GH, Loomis S, Fisher M. Laryngeal reconstruction in children. Assessment of vocal quality. Arch Otolaryngol Head Neck Surg. 1993; 119:504-507.

4. Clary RA, Pengilly A, Bailey M. Analysis of voice outcomes in pediatric patients following surgical procedures for laryngotracheal stenosis. Arch Otolaryngol Head Neck Surg. 1996; 122:1189-1194).

5. Hillel AT, Karatayli-Ozgursoy S, Benke JR, Best S, Pacheco-Lopez P, Teets K, Starmer H, Akst LM. Voice quality in laryngotracheal stenosis: impact of dilation and level of stenosis. Annals of Otology, Rhinology \& Laryngology. 2015 May;124(5):413-8.

6. Smith and Thomson (2013). Influence of subglottic stenosis on the flow-induced vibration of a computational vocal fold model. Journal of Fluids and Structures 38,77-91.

7. Hirano, M. (1981). The clinical examination ofvoice (pp. 4-7). Vienna: Springer-Verlag.

8. Titze, I. R. (1988). The physics of small-amplitude oscillation of the vocal folds. Journal of the Acoustical Society of America, 83, 1536-52.

9. Boessenecker A., Berry D. A., Lohscheller J., Eysholdt U., and Döllinger M. (2007). "Mucosal wave properties of a human vocal fold," Acta Acust. Acust. 93, 815-823.10.

10. Smith S.L., Thomson S.L. (2012). Effect of inferior angle on the self-oscillation of a computational vocal fold model. Journal of Acoustical Sociaety of Americal 131 (5), 4062-4075.

11. Li S., Scherer R. C. , Wan M., Wang S., Wu H. (2006) Numerical study of the effects of inferior and superior vocal fold surface angles on vocal fold pressure distributions. Journal of the Acoustical Society of America, 119:3003-3010.

12. Grisel J., Khosla S., Murugappan S., Lakhamraju R., Aubry J., Gutmark E., Huntress G. (2010). How does the absence or presence of subglottal medialization affect glottal airflow? Annals of Otology, Rhinology and Laryngology, 119 (8):559-566.

13. Bryans L., Palmer A.D., Schindler J.S., Andersen P.E., Cohen J.I. (2013). Subjective and objective parameter of the adult female voice after cricotracheal resection and dilation. Ann Otol Rhino Laryngol. 122 (11):707716. 
14. Hseu A.F., Benninger M.S., Haffey T.M., Lorenz R. (2014). Subglottic stenosis: a ten-year review of treatment outcomes. Laryngoscope, 124:736-741.

15. Hillel A.T., Karatayli-Ozgursoy S., Benke J.R., Best S., Pacheco-Lopez P., Teets K., Starmer H.,
Akst L.M. (2015).Voice quality in laryngotracheal stenosis: impact of dilation and level of stenosis. Ann Otol Rhinol Laryngol.; 124(5):413-8. doi: 10.1177/0003489414564249. 